\title{
Análise da percepção sobre assentos de trabalho utilizando técnicas estatísticas multivariadas
}

\author{
Liane Werner, M.Eng. \\ Departamento de Estatística \\ Universidade Federal do Rio Grande do Sul \\ E-mail:werner@mat.ufrgs.br \\ Júlio Carlos de Souza van der Linden, M.Eng. \\ E-mail: jlinden@ppgep.ufrgs.br \\ José Luis Duarte Ribeiro, Dr. \\ Programa de Pós-Graduação em Engenharia de Produção \\ Universidade Federal do Rio Grande do Sul \\ E-mail: ribeiro@ufrgs.br
}

\begin{abstract}
Resumo
A busca por métodos e ferramentas que permitam avaliar e selecionar assentos para uso em determinadas situações de trabalho tem sido uma preocupação constante no campo da Ergonomia. Neste estudo, foram aplicadas técnicas multivariadas para a análise dos dados provenientes de questionários de desconforto e de satisfação, utilizados para a avaliação de assentos em um experimento realizado em um laboratório industrial. O presente artigo tem como objetivo selecionar o assento percebido como o melhor. Para tanto, fez-se uso da Análise de Variância, da Análise de Aglomerados e da Análise Fatorial. 0 uso dessas técnicas permitiu gerar uma descrição que explica esta seleção através dos construtos obtidos: Bem-Estar, Funcionalidade e Desconforto.
\end{abstract}

\section{Palavras-chave}

Análise multivariada; análise de aglomerados; análise fatorial; conforto; desconforto; assento de trabalho.

\section{Analysis of work seats user's perception by means of statistical multivariate tools}

\begin{abstract}
Methods and tools that allow seat evaluation and selection in specific work environments are permanently a research issue in Ergonomics. In this study, multivariate tools were used to analyse satisfactions and discomfort questionnaires data from a seat evaluation experiment, performed at an industrial laboratory. The aim of this paper is the selection of the best seat based on user's perceptions. Thus, Analysis of Variance, Cluster Analysis, and Factorial Analysis were used to explain the selection based on the obtained constructs: Well-being, Functionality, and Discomfort.
\end{abstract}

Key words

Multivariate analysis; custer analysis; factorial analysis; comfort; discomfort; work seat. 


\section{INTRODUĈ̣̃O}

A busca por métodos e ferramentas que permitam avaliar e selecionar assentos (cadeiras, bancos, etc.) para uso em determinadas situações de trabalho tem sido uma preocupação constante no campo da Ergonomia. Shackel et al. (1969) já apresentam um protocolo de avaliação de assentos baseado em uma única variável: o eixo desconforto/conforto.

A dificuldade na avaliação de assentos começa com a falta de uma definição geral para o conforto, posto que este é subjetivo, dependente em grande parte da percepção da pessoa que está vivenciando a situação. Para o senso comum, e para alguns autores, o conforto é a ausência de desconforto (GOONETILLEKE, 1998). Contudo, estudos recentes propõem que o desconforto é uma entidade independente do conforto (STRACKER,1999; GOONETILLEKE, 1998). Zhang et al. (1996) definem que o conforto trata-se de um sentimento de relaxamento e bem-estar, associado a questões estéticas, enquanto que o desconforto está ligado ao atendimento de questões biomecânicas.

Atualmente, diversos métodos para avaliação de assentos são apresentados na literatura, dividindo-se em dois grandes grupos: avaliações em laboratório e experimentos em condições reais. Além disso, os métodos propostos também se distinguem quanto ao enfoque: $i$ ) objetivos, utilizando medições biomecânicas e fisiológicas ou protocolos de avaliação de mudanças posturais; e ii) subjetivos, baseados em questionários de satisfação ou avaliações psicofísicas.

De uma forma ou de outra, a complexidade da análise dos dados coletados tem aumentado diante do número de variáveis que vêm sendo incorporadas a esses estudos. Assim, a aplicação de técnicas de análise estatística multivariada tem sido uma necessidade para pesquisadores que buscam aprofundar a compreensão dos dados coletados.

Em avaliações de conforto em assentos sob o enfoque psicofísico, Hellander; Zhang (1997) utilizaram a Análise Fatorial, com o objetivo de validar o modelo de conforto/desconforto proposto por Zhang et al. (1996). Outra aplicação sob o mesmo enfoque pode ser encontrada em Fogleman; Lewis (2002), que identificaram fatores de risco associados ao desconforto músculo-esquelético em usuários de terminais de vídeo, com Análise Fatorial. Vergara; Page (2002), estudando o conforto sob a abordagem biomecânica, utilizaram Análise Fatorial para o tratamento inicial das variáveis, por meio de redução, que posteriormente foram analisadas por Regressão Logística e Análise Discriminante.

Neste contexto, a equipe do Núcleo de Design e Ergonomia do Laboratório de Otimização de Produtos e Processos do PPGEP/UFRGS propôs recentemente um método para avaliação de assentos de trabalho (GUIMARÃES et al., 2001a) que incorpora a avaliação psicofísica de desconforto e a avaliação de satisfação em relação a diversas variáveis, entre elas conforto. Esse método foi aplicado para a seleção de assentos em ambientes industriais e administrativos. A primeira aplicação do método envolveu a seleção de assentos adequados ao uso em laboratório de uma empresa de tintas industriais (GUIMARÃES et al., 2001b; GUIMARÃES et al. 2002). Contudo, os dados foram tratados apenas com técnicas estatísticas univariadas e, embora os resultados tenham correspondido à expectativa dos pesquisadores, restaram algumas lacunas que podem ser preenchidas com o enfoque multivariado. Entre elas, o fato de não ter sido atingido o objetivo de discriminar, entre os assentos, aquele com melhor desempenho em relação ao conjunto de variáveis estudadas, de acordo com a percepção dos usuários.

\section{A busca por métodos e ferramentas que para uso em determinadas situações de trabalho tem sido uma preocupação constante no campo da Ergonomia.}

Diante disso, este estudo visa analisar os referidos dados por meio da aplicação de um conjunto de técnicas de análise estatística multivariada, com o objetivo de selecionar o assento percebido como o melhor e, adicionalmente, gerar uma descrição que explique esta seleção.

\section{DESCRICÃO DO CASO}

O estudo a ser analisado neste trabalho foi realizado em um laboratório de tintas industriais com o objetivo de avaliar e selecionar assentos para trabalho que pudessem substituir o banco de madeira então utilizado pelos funcionários. Esse estudo está apresentado em Guimarães et al. (2001b). No laboratório em questão, são realizados testes em amostras de tintas e de concentrados. Parta tanto, os funcionários utilizam postos de traba- 
lho individuais em bancadas, além de cabines de pintura. Também faz parte do trabalho a liberação de ordem de produção, a atualização do sistema, a confecção de padrões para laboratórios e o atendimento a reclamações de clientes. Durante todo o expediente de trabalho ocorrem deslocamentos pelo interior da área de produção. O trabalho apresenta características de um trabalho dinâmico, sendo também um caso típico de trabalho com alternância de posturas em pé e sentado.

Foram avaliados dois tipos de assentos: cadeira alta e banco em pé/sentado, cada qual com três modelos. As três cadeiras altas são variações de um mesmo conceito, tendo os mesmos dispositivos e diferenciando-se apenas pelos materiais utilizados para o assento e o encosto. Conforme pode ser visto na Figura 1, o assento A é revestido de couro sintético, o assento $\mathrm{C}$ é conformado em poliuretano expandido e o assento E é em madeira compensada. Já os bancos em pé/sentado correspondem a três conceitos diversos: o assento $\mathrm{B}$ em poliuretano expandido, montado sobre uma estrutura tubular, com altura regulável; o assento $\mathrm{D}$, montado sobre uma estrutura tubular articulada, com altura regulável; e o assento F em poliuretano expandido, montado sobre um pistão pneumático, com regulagem contínua.

Para realizar a coleta de dados, foi utilizado um projeto experimental, no formato de Quadrado Latino, no qual foram aplicados simultaneamente o Questionário de Satisfação quanto ao uso dos assentos (QS) e o Questionário de Desconforto de partes do corpo (QD), que serão descritos posteriormente. Este experimento foi realizado ao longo de seis dias consecutivos de trabalho, onde cada um dos seis voluntários testou todos os seis assentos, conforme o Quadro 1.

O QS foi aplicado ao final de cada dia do experimento. O QD foi aplicado ao início e ao final de cada dia do experimento, com o objetivo de obter a medida da diferença entre o desconforto sentido no início e no final do dia. Atribui-se essa diferença ao desconforto sentido durante o dia de trabalho.

\section{ANÁLISE DO CASO}

Os dados coletados com a aplicação do QS foram tabulados em um arquivo SPSS, pacote estatístico utilizado para proceder às análises. Da mesma forma, os dados iniciais e finais do QD foram lançados em outro arquivo SPSS, de onde foram gerados os valores das diferenças.

Inicialmente, os dados coletados foram analisados separadamente, conforme apresentado a seguir, nos textos que tratam do questionário de desconforto (QD) e do questionário de satisfação (QS). Posteriormente, os dois

\section{Figura 1: Fotos dos seis assentos do estudo.}

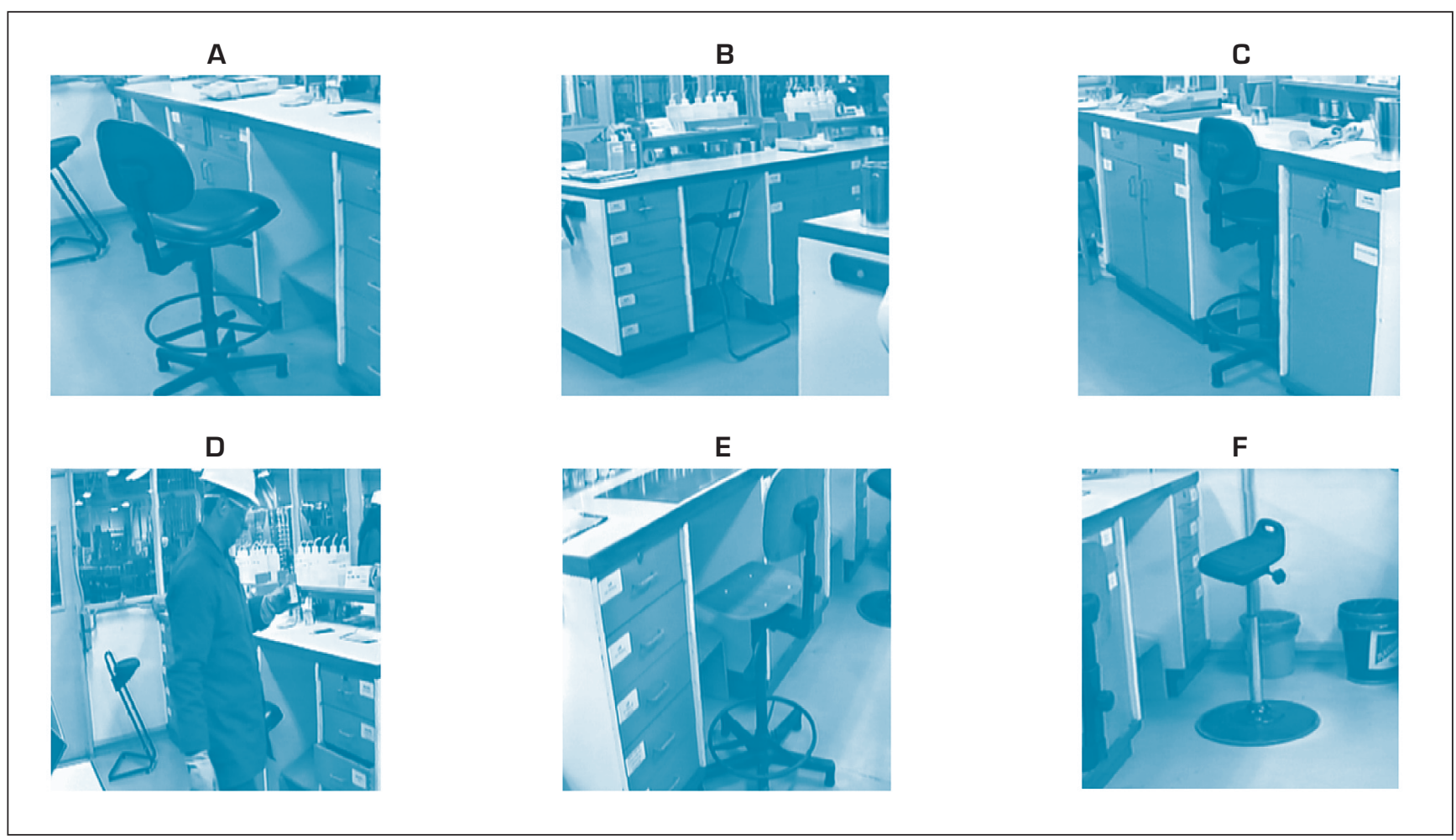


questionários foram analisados em conjunto, com o uso de Análise de Aglomerados e Análise Fatorial.

\section{Análise dos resultados do questionário de desconforto}

O Questionário de Desconforto de partes do corpo (QD) é uma adaptação do instrumento desenvolvido por Corlett; Bishop (1976) e tem o objetivo de medir a ocorrência de desconforto em partes localizadas do corpo. Consiste de uma folha com um mapa corporal dividido em 28 partes. Utiliza uma escala visual análoga de 9 $\mathrm{cm}$, que permite a coleta de dados contínuos, para cada parte do corpo. Para melhor compreensão, um modelo do questionário é apresentado no Anexo 1.

Assume-se que diferenças positivas indicam um aumento do desconforto sentido ao longo de cada dia do experimento. As diferenças negativas indicariam a diminuição no desconforto sentido. Essas diferenças também poderiam ser atribuídas ao erro experimental (falta de acurácia do respondente; fatores não-controlados, como o uso de medicamentos, por exemplo).

Os dados referentes aos 36 casos (assento x indivíduo, uma vez que cada um dos seis assentos foi experimentado por seis indivíduos), relacionados com as 28 variáveis resposta (desconforto de cada parte do corpo), geraram uma matriz de 36 × 28. Para este estudo, foram utilizadas as médias aritméticas das diferenças correspondentes às 28 partes do corpo para cada um dos 36 casos. A média aritmética da variação de desconforto foi assumida como o indicador do desconforto associado ao uso dos assentos para cada indivíduo, gerando a variável Desconforto.

Com o objetivo de verificar a existência de diferenças no desconforto entre os seis assentos, foi procedida uma Análise de Variância Univariada (ANOVA). Nesta análise, os fatores Voluntário e Dia foram considerados com o fim de reduzir a variabilidade residual. Os resultados da ANOVA, apresentados na Tabela 1, demonstram que os efeitos dos fatores Dia e Voluntário para a variável Desconforto foram significativos, conforme era esperado, contudo, a sua análise foge aos objetivos deste trabalho. Optou-se por considerar significativo o efeito do fator Assento ao nível de significância de $10 \%$, na medida em que este experimento foi realizado em situação real de trabalho, sem efetivo controle por parte dos pesquisadores. Dessa forma pode-se proceder à análise das médias da variável por assento apresentadas na Tabela 2.

Quadro 1: Desenho do experimento: as letras A a F referem-se aos diferentes modelos de assento.

\begin{tabular}{|c|c|c|c|c|c|c|}
\hline \multirow[b]{2}{*}{ VOLUNTÁRIOS } & \multicolumn{6}{|c|}{ DIA DO EXPERIMENTO } \\
\hline & 10 & $2^{0}$ & 30 & $4^{0}$ & $5^{0}$ & $\mathbf{G}^{0}$ \\
\hline $\mathbf{I}$ & A & $\mathrm{B}$ & C & $\mathrm{D}$ & $E$ & $\mathrm{~F}$ \\
\hline II & $\mathrm{F}$ & A & B & C & D & $\mathrm{E}$ \\
\hline III & $E$ & $\mathrm{~F}$ & $A$ & B & C & D \\
\hline IV & $\mathrm{D}$ & $\mathrm{E}$ & $\mathrm{F}$ & $A$ & $\mathrm{~B}$ & C \\
\hline $\mathbf{v}$ & $\mathrm{C}$ & $\mathrm{D}$ & $E$ & $\mathrm{~F}$ & A & $\mathrm{B}$ \\
\hline VI & $\mathrm{B}$ & $\mathrm{C}$ & $\mathrm{D}$ & $E$ & $\mathrm{~F}$ & A \\
\hline
\end{tabular}

Tabela 1: Análise de Variância.

\begin{tabular}{|l|c|c|c|c|c|}
\hline \multicolumn{1}{|c|}{ FONTES DE VARIAÇÃO } & SQ & GDL & MQ & F & p-value \\
\hline Assento & 16,59 & 5 & 3,32 & 2,21 & 0,09 \\
\hline Dia & 30,79 & 5 & 6,16 & 4,10 & 0,01 \\
\hline Voluntário & 25,39 & 5 & 5,08 & 3,38 & 0,02 \\
\hline Residual & 30,01 & 20 & 1,50 & & \\
\hline TOTAL & 102,79 & 35 & & & \\
\hline
\end{tabular}


Os valores das médias para a variável Desconforto referentes a cada assento indicam que os assentos $\mathrm{C}, \mathrm{F}$ e A tendem a apresentar, nessa ordem, menor desconforto. Para os assentos D, E e B, as médias indicam a percepção de maior desconforto.

\section{Análise dos resultados do questionário de satisfação}

A satisfação geral do usuário quanto a uso de cada assento é medida por meio do Questionário de Satisfação, que inclui seis critérios de avaliação. A sua concepção baseou-se em um conjunto de critérios para avaliação de assentos de trabalho, apresentados por Grieco et al. (1997), dos quais foram selecionados os seguintes: conforto, segurança, adaptabilidade e praticidade. Além desses, o questionário foi complementado com dois critérios considerados também pertinentes: aparência e adequação ao trabalho. As perguntas são apresentadas com uma escala contínua de $15 \mathrm{~cm}$, que mede o grau de satisfação com relação a cada um dos critérios avaliados, tendo nos extremos as âncoras "pouco satisfeito" e "muito satisfeito". Neste estudo, os usuários responderam ao final de cada dia do experimento, referindo-se ao assento utilizado, às seguintes questões:

- Pensando que um assento deve ser confortável, você está: [pouco satisfeito/muito satisfeito];

- Pensando que um assento deve ser seguro, você está: [pouco satisfeito/muito satisfeito];
- Pensando que um assento deve ser adaptável, você está: [pouco satisfeito/muito satisfeito];

- Pensando que um assento deve ser prático, você está: [pouco satisfeito/muito satisfeito];

- Pensando que um assento deve ser adequado ao seu trabalho, você está: [pouco satisfeito/muito satisfeito];

- Pensando na aparência, você está: [pouco satisfeito/ muito satisfeito].

A Tabela 3 apresenta as médias das variáveis de satisfação para os assentos experimentados, indicando que o assento A foi percebido como o de melhor desempenho para todas as variáveis. Em segundo lugar destacaram-se os assentos C e E. Por fim, os assentos B, D e F foram percebidos como tendo um desempenho semelhante, em média.

Os dados foram submetidos ao teste de MANOVA com todas as variáveis do estudo em relação ao fator assento (One-way MANOVA). A aplicação da MANOVA revelou que existem diferenças significativas entre as variáveis de resposta, conforme apresentado na Tabela 4.

Diante desses resultados, realizou-se a Análise de Variância Univariada (ANOVA) para as variáveis respostas do estudo, com o fim de identificar quais variáveis são responsáveis pelas diferenças. Na Tabela 5 pode-se verificar que todas as variáveis, com exceção da variável "adaptabilidade", são influenciadas significativamente pelo fator assento.

Tabela 2: Médias da variável desconforto por assento.

\begin{tabular}{|c|c|c|c|c|c|c|}
\hline & \multicolumn{7}{|c|}{ ASSENTO } \\
\cline { 2 - 7 } & A & B & C & D & E & F \\
\hline Desconforto & $-0,06$ & 0,65 & $-0,95$ & 0,82 & 0,69 & $-0,60$ \\
\hline
\end{tabular}

Tabela 3-2: Médias das variáveis avaliadas pelo questionário de satisfação por assento

\begin{tabular}{|c|c|c|c|c|c|c|c|}
\hline \multirow[b]{2}{*}{ VARIÁVEL } & \multicolumn{7}{|c|}{ ASSENTO } \\
\hline & A & B & c & D & E & $\mathbf{F}$ & MÉDIA DA VARIÁVEL \\
\hline Conforto & 13,75 & 2,18 & 8,18 & 2,13 & 5,33 & 1,87 & 5,57 \\
\hline Adaptabilidade & 13,83 & 3,12 & 10,52 & 3,93 & 11,75 & 3,47 & 7,77 \\
\hline Segurança & 11,45 & 5,55 & 7,58 & 5,63 & 9,02 & 5,33 & 7,43 \\
\hline Praticidade & 11,53 & 4,65 & 5,98 & 4,70 & 5,73 & 4,08 & 6,11 \\
\hline Adequação ao trabalho & 11,42 & 2,73 & 6,57 & 2,63 & 6,85 & 4,82 & 5,84 \\
\hline Aparência & 13,68 & 2,68 & 12,13 & 2,02 & 5,45 & 2,37 & 6,39 \\
\hline Médias dos assentos & 12,61 & 3,49 & 8,49 & 3,51 & 7,36 & 3,66 & \\
\hline
\end{tabular}


Para as variáveis que apresentaram diferenças significativas, procedeu-se à comparação múltipla de médias. Primeiramente aplicou-se o teste de Scheffé, que é o mais conservador com relação ao erro Tipo I, segundo Hair et al. (1998). Contudo, este procedimen- to não conseguiu captar algumas das diferenças existentes. Desta forma, optou-se por utilizar o Teste de Tukey HSD, que de acordo com Hair et al. (1998) é o seguinte na ordem de indicação. Os resultados deste teste foram sumarizados no Quadro 2. Pode-se con-

Tabela 4: MANOVA para o questionário de satisfação com variável controle: Assento.

\begin{tabular}{|l|c|c|c|c|c|}
\hline \multicolumn{5}{|c|}{ Fator: Assento Teste Multivariado de Significância (S = 5, M = 0, N = 11,5) } \\
\hline TESTE & VALOR & APROX. F & GDL TRAT. & GDL ERRO & SIG. DE F \\
\hline Pillais & 1,79 & 2,69 & 30 & 145 & 0,00 \\
\hline Hotellings & 8,59 & 6,70 & 30 & 117 & 0,00 \\
\hline Wilks & 0,04 & 4,36 & 30 & 102 & 0,00 \\
\hline Roys & 0,87 & & & & \\
\hline
\end{tabular}

Tabela 5: ANOVA para as variáveis do questionário de satisfação com variável controle: Assento.

\begin{tabular}{|l|c|c|c|c|c|c|}
\hline \multicolumn{7}{|c|}{ Fator: Assento Teste F univariado com (5;30) graus de liberdade. } \\
\hline VARIÁVEIs & $\mathbf{S a T r}$ & $\mathbf{S a E}$ & $\mathbf{O M T r}$ & $\mathbf{0 M E}$ & $\mathbf{F}$ & SIG. F \\
\hline Conforto & 665,22 & 179,07 & 133,04 & 5,97 & 22,29 & 0,00 \\
\hline Segurança & 690,24 & 373,99 & 138,05 & 12,47 & 11,07 & 0,00 \\
\hline Adaptabilidade & 183,22 & 735,89 & 36,64 & 24,53 & 1,49 & 0,22 \\
\hline Praticidade & 229,93 & 510,16 & 45,98 & 17,00 & 2,70 & 0,04 \\
\hline $\begin{array}{l}\text { Adequação } \\
\text { ao trabalho }\end{array}$ & 355,94 & 685,46 & 71,19 & 22,85 & 3,12 & 0,02 \\
\hline Aparência & 816,23 & 225,25 & 163,25 & 7,51 & 21,74 & 0,00 \\
\hline
\end{tabular}

Quadro 2: Grupos de diferenças de médias obtidas pelo Teste de Tukey HSD.

\begin{tabular}{|l|c|c|c|c|c|c|c|}
\hline \multirow{2}{*}{\multicolumn{1}{c|}{ VARIÁVEL }} & \multicolumn{7}{c|}{ ASSENTO } \\
\cline { 2 - 8 } & A & B & G & D & E & F & OBS \\
\hline Conforto & $\mathrm{G} 1$ & $\mathrm{G} 3$ & $\mathrm{G} 2$ & $\mathrm{G} 3$ & $\mathrm{G} 2, \mathrm{G} 3$ & $\mathrm{G} 3$ & $\mathrm{~A} \neq \mathrm{B}, \mathrm{C}, \mathrm{D}, \mathrm{E}, \mathrm{F} \quad \mathrm{C} \neq \mathrm{B}, \mathrm{D}, \mathrm{F}$ \\
\hline Adaptabilidade & $\mathrm{G} 1$ & $\mathrm{G} 1$ & $\mathrm{G} 1$ & $\mathrm{G} 1$ & $\mathrm{G} 1$ & $\mathrm{G} 1$ & \\
\hline Segurança & $\mathrm{G} 1$ & $\mathrm{G} 2$ & $\mathrm{G} 1$ & $\mathrm{G} 2$ & $\mathrm{G} 1$ & $\mathrm{G} 2$ & $\mathrm{~A}, \mathrm{C}, \mathrm{E} \neq \mathrm{B}, \mathrm{D}, \mathrm{F}$ \\
\hline Praticidade & $\mathrm{G} 1$ & $\mathrm{G} 1, \mathrm{G} 2$ & $\mathrm{G} 1, \mathrm{G} 2$ & $\mathrm{G} 1, \mathrm{G} 2$ & $\mathrm{G} 1, \mathrm{G} 2$ & $\mathrm{G} 2$ & $\mathrm{~A} \neq \mathrm{F}$ \\
\hline Adequação ao trabalho & $\mathrm{G} 1$ & $\mathrm{G} 2$ & $\mathrm{G} 1, \mathrm{G} 2$ & $\mathrm{G} 2$ & $\mathrm{G} 1, \mathrm{G} 2$ & $\mathrm{G} 1, \mathrm{G} 2$ & $\mathrm{~A} \neq \mathrm{B}, \mathrm{D}$ \\
\hline Aparência & $\mathrm{G} 1$ & $\mathrm{G} 2$ & $\mathrm{G} 1$ & $\mathrm{G} 2$ & $\mathrm{G} 2$ & $\mathrm{G} 2$ & $\mathrm{~A}, \mathrm{C} \neq \mathrm{B}, \mathrm{D}, \mathrm{E}, \mathrm{F}$ \\
\hline
\end{tabular}


cluir, com base na análise dos grupos formados, que existe uma tendência de o assento A diferir de o assento F. Os assentos A e C diferem apenas para a variável Conforto, apresentando similaridade em relação aos demais aspectos. Além disso, percebem-se também diferenças entre o assento A e os assentos B e D.

uma escala contínua com variação de 0 a 15 . Por seu lado, as respostas para desconforto foram geradas a partir da diferença entre valores que poderiam variar, também em escala contínua, entre 0 e 9. Nesse caso, fez-se a opção de padronizar as respostas com a utilização da variável reduzida $Z$. As médias dos resultados padronizados são apresentadas na Tabela 6 .

Com base nos dados padronizados foi feita a Análise de Aglomerados preliminar. No primeiro momento utilizou-se a técnica hierárquica de Análise de Aglomerados, com o método que se baseia na distância entre os centróides dos grupos formados. Por meio da inspeção do dendograma, que se encontra na Figura 2, é possível identificar dois

Na medida em que os resultados desta análise e da anterior não permitiram estabelecer o assento ou grupo de assentos que se mostrem mais adequados às expectativas dos usuários, decidiu-se proceder a outros tipos de análise com os dados dos dois questionários associados.

\section{Utilização da Análise de Aglomerados para estudar o desempenho dos assentos}

Com base na associação dos resultados dos dois questionários buscou-se encontrar agrupamentos que pudessem explicar o desempenho dos assentos, considerando as variáveis Conforto, Segurança, Adaptabilidade, Praticidade, Adequação ao Trabalho, Aparência e Desconforto. Para tanto, os dados foram reorganizados e tratados para permitir o uso das técnicas de Análise de Aglomerados.

O exame dos dados demonstrou a necessidade de padronizar as escalas das variáveis a serem submetidas à Análise de Aglomerados. As respostas referentes ao grupo de variáveis do questionário de satisfação utilizam grupos principais, um formado predominantemente pelos assentos A, C e E e o outro pelos assentos B, D e F. Contudo, formou-se também um terceiro agrupamento, que analisado mais detalhadamente reflete a percepção do voluntário IV.

Foi utilizada, em seguida, a técnica de Análise de Aglomerados não-hierárquica. Fez-se a opção de formar 3 e 4 agrupamentos, para verificar outros agrupamentos que permitissem, ou não, reforçar as observações feitas a partir do dendograma. O Quadro 3 apresenta os resultados da formação de três agrupamentos. Pode-se observar um comportamento semelhante ao verificado por meio da Análise Hierárquica. O agrupamento 3 capta a percepção dos voluntários em relação aos assentos B, D e F. Por sua vez, o mesmo acontece com o agrupamento 2 com relação ao assento A. A percepção com relação aos assentos $\mathrm{C}$ e E é difusa entre os três agrupamentos. O agrupamento 1 não está associado a um determinado assento, sendo dominado pela percepção do voluntário IV.

Tabela 6: Médias das variáveis padronizadas por assento, incluindo desconforto.

\begin{tabular}{|c|c|c|c|c|c|c|}
\hline \multirow[b]{2}{*}{ VARIÁVEL } & \multicolumn{6}{|c|}{ ASSENTO } \\
\hline & A & B & c & D & E & $\mathbf{F}$ \\
\hline Conforto & 1,67 & $-0,71$ & 0,53 & $-0,70$ & $-0,05$ & $-0,74$ \\
\hline Adaptabilidade & 0,80 & $-0,37$ & 0,03 & $-0,35$ & 0,31 & $-0,41$ \\
\hline Segurança & 1,10 & $-0,84$ & 0,50 & $-0,69$ & 0,72 & $-0,78$ \\
\hline Praticidade & 1,19 & $-0,35$ & $-0,03$ & $-0,30$ & $-0,08$ & $-0,44$ \\
\hline Adequação ao trabalho & 0,97 & $-0,62$ & 0,08 & $-0,64$ & 0,44 & $-0,24$ \\
\hline Aparência & 1,34 & $-0,68$ & 1,05 & $-0,82$ & $-0,17$ & $-0,72$ \\
\hline Desconforto & $-0,10$ & 0,33 & $-0,61$ & 0,43 & 0,35 & $-0,39$ \\
\hline
\end{tabular}


Figura 2: Dendograma das variáveis de satisfação e desconforto para os seis assentos.

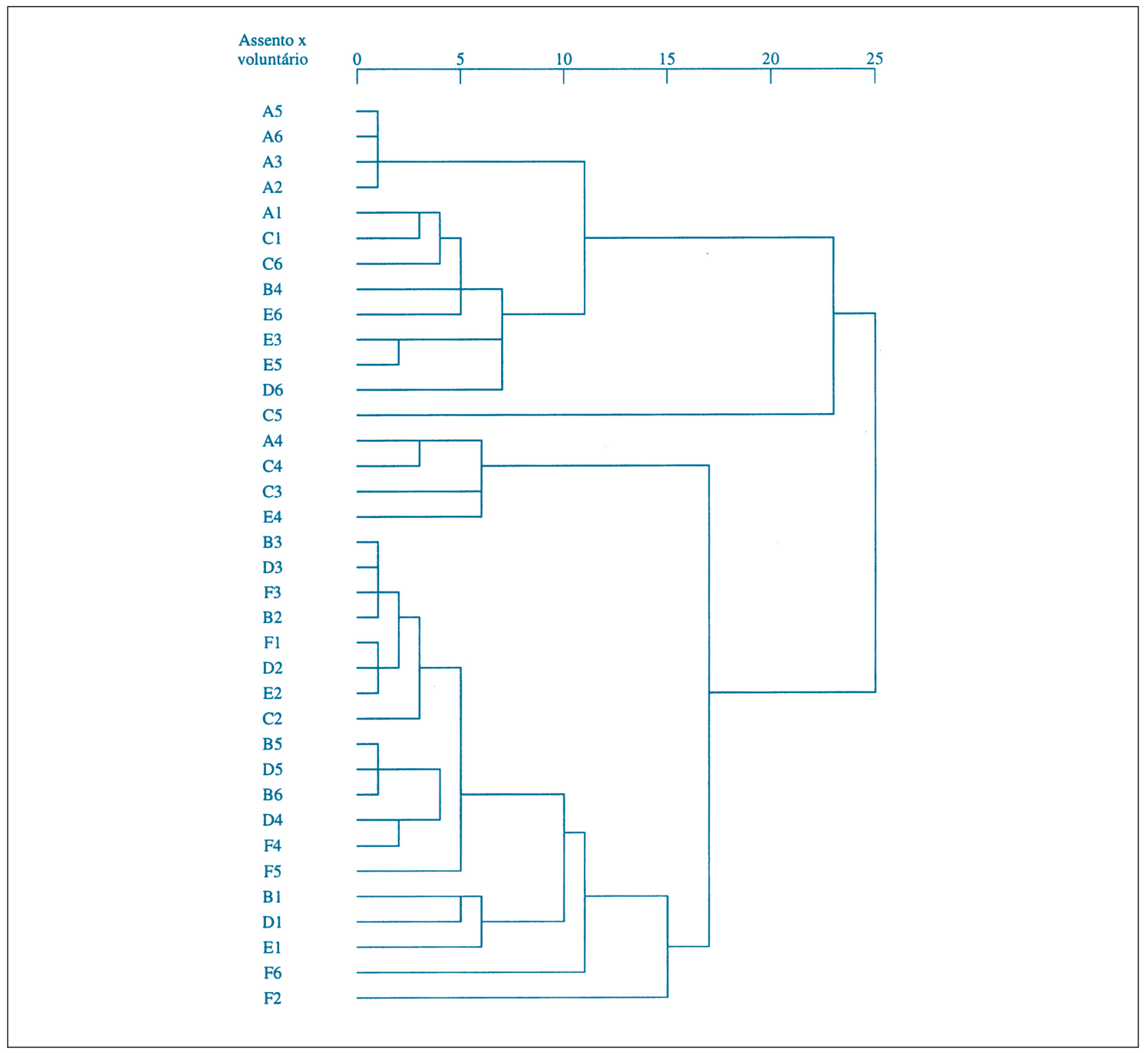

Quadro 3: Agrupamentos por voluntário x assento gerados pela Análise de Aglomerados (três grupos).

\begin{tabular}{|c|c|c|c|c|c|c|}
\hline \multirow[b]{2}{*}{ VOLUNTÁRIOS } & \multicolumn{6}{|c|}{ ASSENTO } \\
\hline & $\mathbf{A}$ & $\mathbf{B}$ & C & $\mathbf{D}$ & $\mathbf{E}$ & $\mathbf{F}$ \\
\hline I & 2 & 3 & 2 & 3 & 1 & 3 \\
\hline II & 2 & 3 & 3 & 3 & 3 & 3 \\
\hline III & 2 & 3 & 1 & 3 & 2 & 3 \\
\hline IV & 1 & 2 & 1 & 3 & 1 & 3 \\
\hline $\mathrm{V}$ & 2 & 3 & 2 & 3 & 2 & 2 \\
\hline $\mathrm{VI}$ & 2 & 3 & 2 & 2 & 2 & 3 \\
\hline
\end{tabular}


A análise realizada com quatro agrupamentos não ofereceu uma melhor interpretação dos resultados. Como pode ser visto no Quadro 4, a mudança substancial se deu na divisão do agrupamento 3 , que gerou neste caso os agrupamentos 3 e 4 . Os quatro agrupamentos resultantes desta análise permitiram caracterizar apenas dois assentos, o A e o F. A percepção em relação aos demais assentos têm seu comportamento difuso em maior ou menor grau. $\mathrm{O}$ agrupamento 1 permanece representando a percepção do voluntário IV.

Nas duas formas de agrupamento não-hierárquico os assentos $\mathrm{C}$ e $\mathrm{E}$ não foram associados a um agrupamento específico, além disso um dos agrupamentos não representa um assento, mas sim está associado à percepção de um voluntário. Assim, optou-se por investigar a percepção sobre os assentos a partir das médias das respostas para cada variável em cada assento (média das respostas de todos os voluntários). Com base nessas médias, foi realizada a Análise de Aglomerados hierárquica com o método da distância entre centróides. A inspeção do dendograma, apresentado na Figura 3, permite identificar três agrupamentos: um formado pelos assentos B, D e
$\mathrm{F}$, outro pelos assentos $\mathrm{C}$ e $\mathrm{E}$ e o último apenas pelo assento A.

Essa última análise confirmou as análises anteriores, de que a percepção geral sobre o assento A é diferenciada dos demais assentos. Com base na Tabela 3, devido a suas altas médias para as seis variáveis de satisfação, fica evidente que o assento A é percebido como o melhor. Esse assento é um dos modelos de cadeira alta, que se diferencia dos demais, $\mathrm{C}$ e E, pelo tipo de material de encosto e assento. Cabe observar ainda que o agrupamento $\mathrm{B}, \mathrm{D}$ e $\mathrm{F}$ corresponde aos bancos em pé/sentado.

Contudo, o uso da Análise de Aglomerados não permitiu explicar de que forma o conjunto de variáveis do estudo gerou a discriminação entre os assentos. Para tanto, tornou-se necessário utilizar outras ferramentas que permitissem descrever a percepção sobre os assentos.

\section{Utilização da Análise Fatorial para identificação dos construtos}

A Análise Fatorial pode ser utilizada tanto com o fim de reduzir o número de variáveis de resposta, em casos nos quais a sua quantidade dificulta a análise e a discus-

Quadro 4: Agrupamentos por voluntário x assento gerados pela Análise de Aglomerados (quatro grupos).

\begin{tabular}{|c|c|c|c|c|c|c|}
\hline \multirow{2}{*}{ VoLUNTÁRIOS } & \multicolumn{7}{|c|}{ ASSENTO } & E & F \\
\cline { 2 - 7 } & A & B & C & D & 3 & 4 \\
\hline I & 2 & 3 & 4 & 3 & 4 & 4 \\
\hline II & 2 & 3 & 3 & 4 & 2 & 3 \\
\hline III & 2 & 3 & 1 & 3 & 1 & 4 \\
\hline IV & 1 & 4 & 1 & 4 & 2 & 4 \\
\hline V & 2 & 4 & 2 & 4 & 2 & 4 \\
\hline
\end{tabular}

Figura 3: Dendograma das médias das variáveis de satisfação e desconforto para os seis assentos.

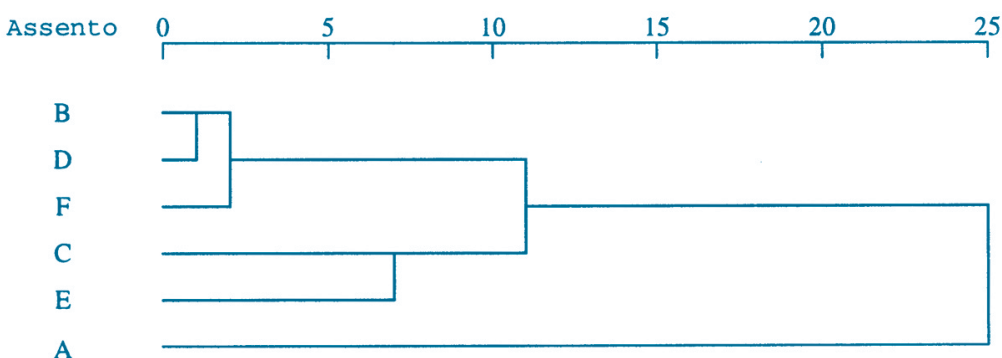


são, como para permitir identificar uma estrutura subjacente a essas variáveis. Neste estudo, a Análise Fatorial foi usada para a identificação de construtos que permitissem caracterizar como os usuários perceberam os diversos critérios durante a avaliação dos assentos.

Antes da realização da análise, foram verificados aspectos críticos, relacionados com pressupostos teóricos, iniciando pela verificação do tamanho da amostra. Para o caso da Análise Fatorial recomenda-se um tamanho de amostra ao redor de, pelo menos, 10 observações por variável, contudo, é aceitável trabalhar com uma relação de 5:1 (HAIR et al., 1998). No caso, a relação entre o número de observações (36) e o número de variáveis (7) atende a esse limite mínimo.

Outro passo antes da realização da Análise Fatorial está relacionado com a suposição da existência de correlações entre variáveis. Uma das maneiras de verificar a adequação dos dados a uma Análise Fatorial é o exame da matriz de correlação, identificando se há correlações significativas. Neste estudo, a matriz da Tabela 7 revela que 15 das 21 correlações são significantes ao nível de $p=0,05$. Também revela que a variável "desconforto" não apresenta correlações significativas com nenhuma das demais variáveis, o que poderia sugerir a sua exclusão desta análise. Contudo, como há o interesse em estudar o seu efeito na percepção geral, essa variável será mantida.

A inspeção da matriz de correlação já indica a tendência à formação de três fatores, com base nas correlações superiores a 0,7 . O primeiro fator seria dominado pelas variáveis Conforto, Segurança e Aparência, enquanto o segundo fator englobaria as variáveis Adaptabilidade, Praticidade e Adequação ao Trabalho. O terceiro fator seria dominado pela variável Desconforto.

Após esta inspeção inicial, foram utilizadas outras estatísticas com o mesmo objetivo: o Teste de Esfericidade de
Bartlett e a medida de Kaiser-Meyer-Olkin (KMO). Para todas essas estatísticas, os resultados indicaram a aceitação do uso da Análise Fatorial para o tratamento dos dados do estudo. O Teste de Esfericidade de Bartlett mede a probabilidade da matriz de correlações apresentar correlações significantes para pelo menos alguma das variáveis. Nesse teste, o resultado obtido foi significante ( $p$-value 0,000$)$. Para a medida de $\mathrm{KMO}$, ou medida de adequação da amostra, na qual valores acima de 0,700 são aceitáveis, foi obtido o valor de 0,750 .

A partir destes procedimentos, foi realizada a extração dos fatores por meio do método de componentes principais. Os resultados iniciais, conforme o critério de seleção de autovalores maiores que 1 , levaram à formação de dois fatores. Como pode ser visto na Tabela 8 , para o $1^{\circ}$ fator, as variáveis Desconforto e Aparência não apresentaram cargas significativas para o tamanho da amostra, enquanto que o $2^{\circ}$ fator não apresentou qualquer variável

Tabela 8: Cargas fatoriais pelo método de componentes principais.

\begin{tabular}{|l|c|c|}
\hline \multirow{2}{*}{\multicolumn{1}{|c|}{ VARIÁVEL }} & \multicolumn{2}{c|}{ FATOR } \\
\cline { 2 - 3 } & $\mathbf{1}$ & $\mathbf{2}$ \\
\hline Conforto & $\mathbf{0 , 8 9 1}$ & $\mathbf{0 , 3 1 3}$ \\
\hline Segurança & 0,861 & 0,323 \\
\hline Adaptabilidade & 0,851 & $-0,346$ \\
\hline Praticidade & 0,829 & $-0,278$ \\
\hline Adequação ao trabalho & 0,881 & $-0,266$ \\
\hline Aparência & 0,759 & 0,505 \\
\hline Desconforto & $-0,343$ & 0,531 \\
\hline
\end{tabular}

Tabela 7: Matriz de correlação de Pearson para as variáveis do estudo.

\begin{tabular}{|c|c|c|c|c|c|c|c|}
\hline & CONFORTO & SEGURANÇA & $\begin{array}{l}\text { ADAPTA- } \\
\text { BILIDADE }\end{array}$ & PRATICIDADE & \begin{tabular}{|l|} 
ADEQUAÇÃOO \\
AO TRABALHO
\end{tabular} & APARÊNCIA & $\begin{array}{l}\text { DESCON- } \\
\text { FORTO }\end{array}$ \\
\hline Conforto & 1,000 & $0,866 *$ & $0,569 *$ & $0,620 *$ & $0,651^{*}$ & $0,828 *$ & $-0,290$ \\
\hline Segurança & $0,866 \%$ & 1,000 & $0,627^{*}$ & $0,535^{*}$ & $0,664^{*}$ & $0,720 *$ & $-0,171$ \\
\hline Adaptabilidade & $0,569 *$ & $0,627^{*}$ & 1,000 & $0,813^{*}$ & $0,860 *$ & $0,436 *$ & $-0,280$ \\
\hline Praticidade & $0,620 *$ & $0,535 *$ & $0,813 *$ & 1,000 & $0,800 *$ & $0,464^{*}$ & $-0,206$ \\
\hline $\begin{array}{l}\text { Adequação } \\
\text { ao trabalho }\end{array}$ & $0,651 *$ & $0,664^{*}$ & $0,860 *$ & $0,800^{*}$ & 1,000 & $0,483^{*}$ & $-0,272$ \\
\hline Aparência & $0,828 *$ & $0,720 *$ & $0,436 *$ & $0,464 *$ & $0,483^{*}$ & 1,000 & $-0,152$ \\
\hline Desconforto &,- 290 &,- 171 &,- 280 &,- 206 &,- 272 &,- 152 & 1,000 \\
\hline
\end{tabular}

* Correlação significante ao nível de 0.05 . 
com carga significativa. A análise desses fatores, considerando a significância das cargas fatoriais, demonstrou que não conduziam a uma explicação adequada. Para essa decisão, foi adotado o critério recomendado por Hair et al. (1998), segundo o qual, por exemplo, para aceitar como significativa uma carga fatorial de 0,50 , o tamanho mínimo da amostra deve ser de 120 observações. A partir desse critério, e na medida em que o tamanho de amostra neste estudo é de 36 observações, foi assumido que uma carga fatorial é significativa quando for superior a 0,8 .

Considerando que a extração pelo critério de autovalores maiores que 1 não apresentou resultado satisfatório, foi procedida a inspeção da Tabela 9 de onde se observou que o terceiro fator contribui em aproximadamente $13 \%$, corroborando as indicações fornecidas pela matriz de correlação. Ao acrescentar o terceiro fator à análise, obteve-se uma explicação de $90 \%$ da variância total.

A decisão de extrair 3 fatores por si não seria suficiente para atender aos objetivos da análise, na medida em que o 2 o fator não apresentou variável com carga signifi- cativa. Diante disso, para a nova extração foi necessário incluir o procedimento de rotação, com a expectativa de obter uma melhor distribuição das cargas. Considerando que o objetivo desta análise fatorial é a obtenção de construtos e não a redução do número de variáveis, optou-se por uma rotação oblíqua, conforme recomendado por Hair et al. (1998). Foi utilizada a rotação oblíqua Direct Oblimin, disponível no programa SPSS, obtendose as cargas fatoriais conforme a Tabela 10. Pode-se observar que os fatores apresentam cargas altas para as variáveis, conforme previamente indicado por meio da inspeção da matriz de correlação.

Em seguida, passou-se à interpretação dos fatores com relação às variáveis do estudo, atribuindo-se a cada fator uma designação que represente seu significado. $O$ fator 1 é dominado pelas variáveis Adaptabilidade, Praticidade e Adequação ao Trabalho, que podem ser aspectos funcionais do assento, compondo assim o construto Funcionalidade. Já o fator 2 apresenta o predomínio das variáveis Conforto, Segurança e Aparência, que estão relacionadas com a percepção de bem-estar, gerando o segundo construto, denominado Bem-Estar. O fator 3, que apre-

Tabela 9: Variância total explicada.

\begin{tabular}{|c|c|c|c|}
\hline FATOR & AUTOVALOR & $\%$ DA VARIÂNGIA & $\%$ DA VARIÂNCIA ACUMULADA \\
\hline 1 & 4,414 & 63,06 & 63,06 \\
\hline 2 & 1,007 & 14,39 & 77,44 \\
\hline 3 & 0,883 & 12,61 & 90,06 \\
\hline 4 & 0,304 & 4,34 & 94,40 \\
\hline 5 & 0,186 & 2,66 & 97,07 \\
\hline 6 & 0,143 & 2,04 & 99,11 \\
\hline 7 & 0,062 & 0,89 & 100,00 \\
\hline
\end{tabular}

Tabela 10: Cargas fatoriais pelo método de rotação Oblimin.

\begin{tabular}{|l|c|c|c|}
\hline \multirow{2}{*}{\multicolumn{1}{|c|}{ VARIÁVEL }} & \multicolumn{3}{c|}{ FATOR } \\
\cline { 2 - 4 } & $\mathbf{1}$ & $\mathbf{2}$ & $\mathbf{3}$ \\
\hline Conforto & 0,101 & $\mathbf{0 , 8 7 9}$ & $-0,093$ \\
\hline Segurança & 0,188 & $\mathbf{0 , 8 0 5}$ & 0,040 \\
\hline Adaptabilidade & $\mathbf{0 , 9 6 0}$ & $-0,033$ & $-0,037$ \\
\hline Praticidade & $\mathbf{0 , 9 3 4}$ & 0,002 & 0,045 \\
\hline Adequação ao trabalho & $\mathbf{0 , 8 8 6}$ & 0,081 & $-0,028$ \\
\hline Aparência & $-0,127$ & $\mathbf{1 , 0 0 6}$ & 0,020 \\
\hline Desconforto & 0,001 & $-0,002$ & $\mathbf{0 , 9 9 9}$ \\
\hline
\end{tabular}


senta carga alta apenas para a variável Desconforto, constituiu o construto Desconforto.

Para interpretar o conjunto de dados deste estudo a partir dos três construtos formados, o próximo passo foi o cálculo dos escores fatoriais para cada caso (assento $\mathrm{x}$ indivíduo). Os escores fatoriais consistem no produto matricial dos valores observados para as variáveis pelas cargas fatoriais. Com base nesses escores, foram obtidas as médias referentes a cada construto para os seis assentos, conforme a Tabela 11.

Para descrever como as variáveis do estudo expressam a percepção dos voluntários em relação aos assentos, por meio dos três construtos, foi utilizada a inspeção visual dos gráficos nas Figuras 4 a 6. Para facilitar a compreensão desses gráficos, foi adicionada aos gráficos uma seta que indica a direção do eixo desejável para cada conjunto de construtos. $\mathrm{O}$ assento ideal deveria apresentar altos escores para os construtos, resultando em um ponto próximo ao eixo desejável.

Conforme o gráfico da Figura 4, observou-se que o assento A tem a melhor projeção no eixo desejável, distanciando-se dos demais. $\mathrm{O}$ assento $\mathrm{C}$ tem uma proje-

Tabela 11: Escores médios de cada construto para os seis assentos.

\begin{tabular}{|c|c|c|c|}
\hline \multirow{2}{*}{ ASSENTO } & \multicolumn{3}{|c|}{ CONSTRUTO } \\
\cline { 2 - 4 } & FUNGIONALIDADE & BEM-ESTAR & DESCONFORTO \\
\hline A & 1,027 & 1,469 & $-0,118$ \\
\hline B & $-0,470$ & $-0,796$ & 0,320 \\
\hline C & $-0,019$ & 0,786 & $-0,594$ \\
\hline D & $-0,435$ & $-0,801$ & 0,430 \\
\hline E & 0,285 & 0,146 & 0,356 \\
\hline F & $-0,388$ & $-0,804$ & $-0,396$ \\
\hline
\end{tabular}

Figura 4: Diagrama de dispersão dos escores fatoriais médios dos seis assentos para os construtos Bem-Estar e Funcionalidade.

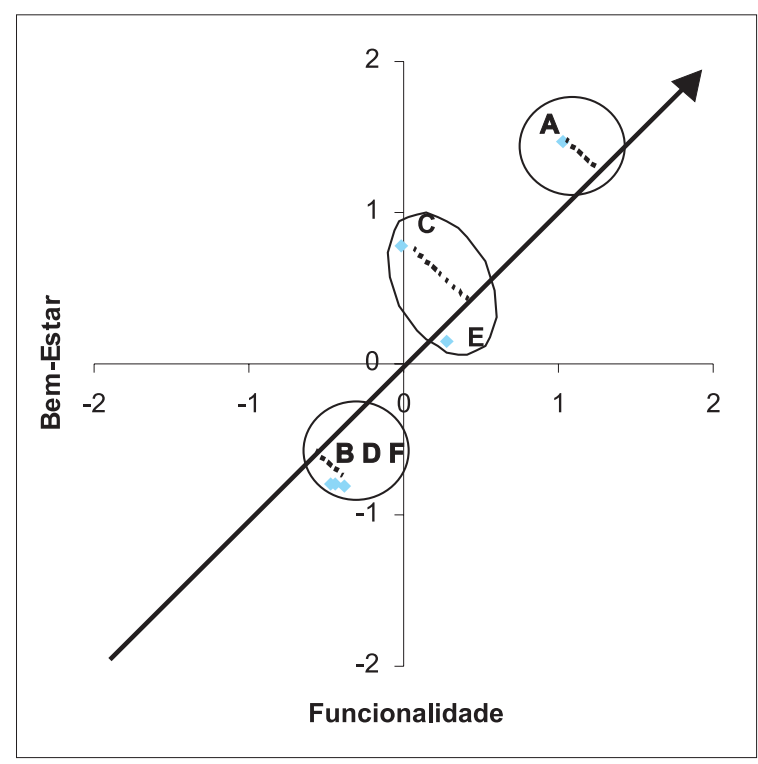


ção levemente superior à do assento E. Embora o assento $\mathrm{C}$ tenha sido melhor percebido quanto ao BemEstar, o assento E é mais equilibrado em relação aos dois construtos. Assim, pode-se interpretar que ambos tiveram um desempenho similar segundo a percepção dos voluntários, tal como encontrado na Análise de Aglomerados. Para os assentos B, D e F, é evidente que foram percebidos da mesma forma, com baixo desempenho para os construtos Bem-Estar e Funcionalidade.

O gráfico da Figura 5 apresenta os construtos BemEstar e Desconforto. Os assentos A e C apresentam projeções próximas ao eixo, indicando desempenhos similares. Contudo, pode-se considerar o assento $\mathrm{C}$ superior, a despeito do escore do assento A para o Bem-Estar, por ser mais equilibrado. Os assentos E e F, apesar de apresentarem projeções próximas, estão em quadrantes opostos, indicando que foram percebidos de forma contrária: $\mathrm{o}$ assento E proporciona mais bem-estar e o assento $\mathrm{F}$ é menos desconfortável. Os assentos D e B foram percebidos da mesma forma, como sendo os mais desconfortáveis e os que causam menor bem-estar.

Com base na premissa de que o aumento do bem-estar está associado a uma redução do desconforto, era de se esperar que o assento A apresentasse um comportamento superior para o construto Desconforto. Considerando que esse assento corresponde ao senso comum de assento confortável (GOONETILLEKE, 1998; van der LINDEN; KUNZLER, 2001), pode-se inferir que a sua aparência afetou favoravelmente a avaliação do construto BemEstar, conforme a Tabela 3. De maneira inversa, para o assento $\mathrm{F}$ era de se esperar uma melhor avaliação para o construto Bem-Estar, devido ao baixo nível que apresentou para o Desconforto, considerando os assentos avaliados. Da mesma forma, pode-se inferir que a sua aparência pouco familiar, como pode ser observado na Figura 1, afetou a avaliação das variáveis que compõem o construto Bem-Estar.

$\mathrm{Na}$ Figura 6, observa-se que o assento A apresenta a melhor projeção sobre o eixo, por conta de ter sido percebido como o de maior funcionalidade. $\mathrm{O}$ assento $\mathrm{C}$ teve a segunda melhor projeção, devido a ter sido o menos desconfortável. Os assentos F e E, novamente, apresentaram projeções próximas, mas estão em quadrantes opostos, indicando que foram percebidos de forma contrária: o assento E proporciona mais funcionalidade e o assento F é menos desconfortável. Novamente, os assentos B e D forma percebidos de forma similar, sendo os de mais baixa funcionalidade e maior desconforto.

Cabe observar ainda, na Figura 6, que a percepção quanto à funcionalidade dos assentos $\mathrm{A}, \mathrm{C}$ e $\mathrm{E}$ não é coerente com as suas características técnicas. Esses assentos são as três cadeiras altas que, conforme descrito

Figura 5: Diagrama de dispersão dos escores fatoriais médios dos seis assentos para os construtos Bem-Estar e Desconforto.

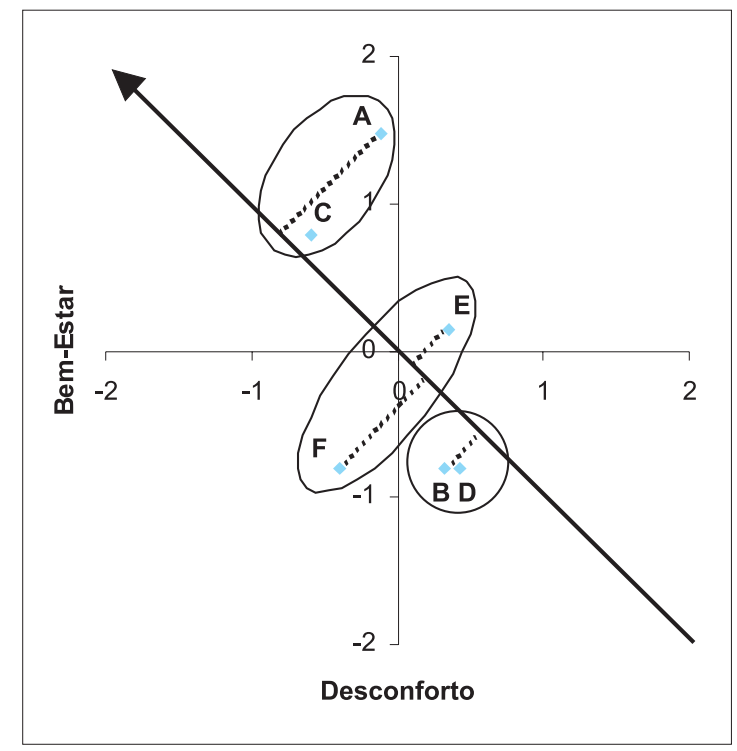


na seção 2, são construídas com os mesmos dispositivos, diferenciando-se apenas quanto aos materiais de assento e encosto. Dessa forma, seria de se esperar uma avaliação com menor variabilidade. Novamente, pode-se inferir que houve um efeito da aparência na avaliação das variáveis que compõem o construto Funcionalidade.

Para finalizar esta análise, salienta-se que o assento A apresentou melhor desempenho para os construtos Bem-Estar e Funcionalidade, enquanto que o assento C destacou-se no construto Desconforto. Os assentos E e F demonstraram um desempenho intermediário e os assentos B e D foram classificados com o pior desempenho.

\section{CONCLUSÕES E CONSIDERAC̣̃̃ES FINAIS}

A aplicação de técnicas de análise multivariada a um conjunto de dados sobre a percepção dos usuários, relativas a diversos assentos de trabalho, permitiu aprofundar as conclusões de Guimarães et al. (2001b), não apenas ao definir qual o assento que melhor atende às necessidades do trabalho em um laboratório de tintas industriais, conforme percebido pelos usuários, como também ao descrever os construtos que explicam essa percepção.

A utilização de Análise de Variância demonstrou que existem diferenças significativas entre os assentos para os critérios de avaliação utilizados, com exceção da
Adaptabilidade. A despeito disso, os resultados da Análise de Variância não permitiram conclusões que viessem a estabelecer um ordenamento entre os assentos.

Quanto ao Questionário de Desconforto, cabe questionar se a obtenção de diferenças significativas apenas ao nível de $10 \%$ decorre de falta de acurácia do instrumento de medição, ou se reflete de fato o que os voluntários sentem. Por um lado, o número de partes do corpo avaliadas pode dificultar o registro do que efetivamente está sendo sentido. Por outro lado, a escala contínua adotada talvez não seja a mais adequada para este tipo de avaliação. Nesse sentido, estudos de Shen; Parsons (1997) demonstram que os resultados de avaliação de desconforto são fortemente dependentes das propriedades da escala.

Com relação aos resultados da MANOVA para o Questionário de Satisfação, cabe ressaltar que talvez a variável Adaptabilidade tenha sido confundida com a variável Praticidade, o que pode ter levado à sua não-significância, como pode ser visto nas declarações dos voluntários em Guimarães et al. (2002). Por isso, recomenda-se que em futuros estudos os pesquisadores certifiquem-se de que os voluntários estão devidamente esclarecidos quanto às diferenças entre essas duas variáveis.

A aplicação da Análise de Aglomerados, permitiu chegar a conclusões com relação aos assentos, discriminando o assento A como aquele melhor percebido pelos voluntários. Contudo, essa análise não explica de que

Figura 6: Diagrama de dispersão dos escores fatoriais médios dos seis assentos para os construtos Funcionalidade e Desconforto.

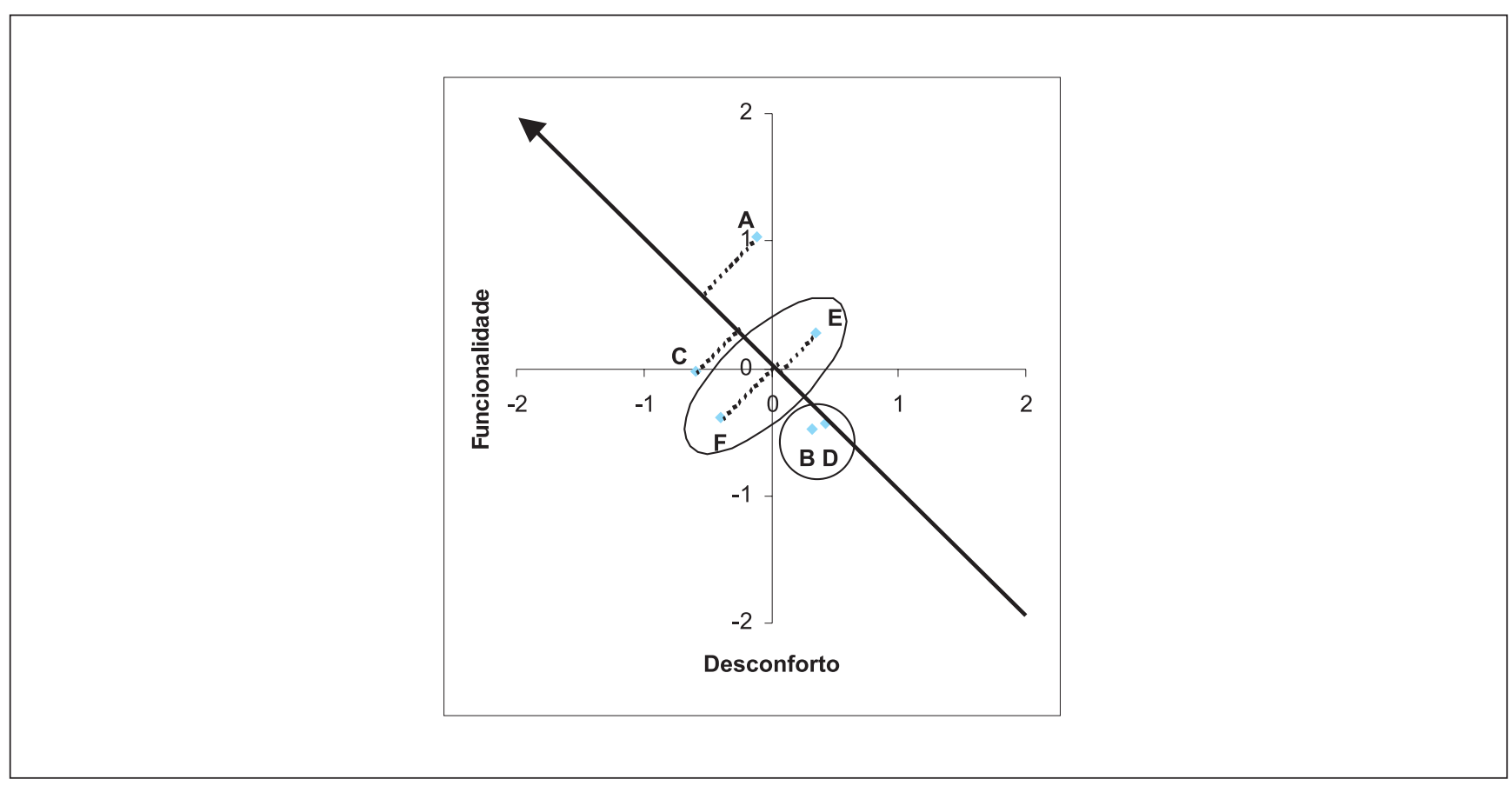


forma o conjunto de variáveis do estudo gerou a discriminação entre os assentos.

Com o uso da Análise Fatorial, foram gerados construtos que permitem explicar de forma sumária a percepção dos voluntários ao avaliarem os assentos. O construto Bem-Estar corrobora a literatura, na medida em que estudos de Zhang et al. (1996) e Goonetilleke (1998) apontam para uma forte correlação entre conforto e estética. Da mesma forma, o construto Desconforto corresponde a estudos que tanto relatam a dificuldade em ser medido de maneira confiável quanto à hipótese de que se trata de uma dimensão isolada (ZHANG et al., 1996; HELANDER; ZHANG, 1997). Por outro lado, a geração do construto Funcionalidade não encontra refe- rências na literatura sobre o assunto de conforto/desconforto em assentos, sendo uma contribuição deste estudo.

Por fim, cabe comentar que a percepção dos voluntários quanto ao construto Funcionalidade para os assentos A, C e E não foi a mesma, a despeito do fato de serem construídos com os mesmos recursos técnicos. Para os construtos Bem-Estar e Desconforto, a diferença é explicável pelos diferentes materiais usados para assento e encosto. O efeito da aparência sobre a percepção pode ser interpretado sob a ótica da Semântica de Produtos, que tem se dedicado a investigar os significados que as pessoas atribuem aos produtos: "o ser humano não responde às qualidades físicas das coisas, mas ao que elas significam para ele" (KRIPPENDORFF, 2000).

\section{Artigo recebido em 13/08/2002 Aprovado para publicação em 29/09/2003}

\section{neferências}

CORLETT, E. N., BISHOP, R.P. A technique for assessing postural discomfort. Ergonomics, v. 19, p. 175$182,1976$.

FOGLEMAN, M., LEWIS, R. J. Factors associated with selfreported musculoskeletal discomfort in video display terminal (VDT) users, International Journal of Industrial Ergonomics, aceito em outubro de 2001 e aguarda impressão. Disponível em http://www.sciencedirect.com, desde 16 de janeiro de 2002 .

GOONETILLEKE, R.S. Design to Minimize Discomfort. Ergonomics in Design, Jul 1998, p. 12 - 19.

GRIECO, A. et al. Criteria for the Ergonomic evaluation of work chair. Proceedings of work with display unities International Scientific Conference, 5 th, 1997. Waseda University, Tokio, Japan, 1997.

GUIMARÃES, L. B. M., FISCHER, D., DINIZ, R., VAN DER LINDEN, J. C. S., KMITA, S., PASTRE, T. Método Macroergonômico para Avaliação de Assentos para Trabalho. In: I Congresso Internacional de
Ergonomia e Usabilidade de Interfaces Humano-Tecnologia: Produtos, Programas, Informação, Ambiente Construído, ERGODESIGN, 2001, Rio de Janeiro. Anais do I Ergodesign. Rio de Janeiro: PUC-Rio,2001a (CD-ROM).

GUIMARÃES, L. B. M., VAN DER LINDEN, J. C. S., FISCHER, D., KMITA, S. Avaliação de assentos de trabalho em laboratório. In: $3^{\circ}$ Congresso Brasileiro de Gestão de Desenvolvimento de Produto, 2001, Florianópolis, SC. Anais do $3^{\circ}$ CBDGP. Florianópolis: UFSC, 2001b (CDROM).

GUIMARÃES, Lia Buarque de Macedo, VAN DER LINDEN, Júlio Carlos de Souza, FISCHER, Daniela, DINIZ, Raimundo, KMITA, Silvério. Que qualidades de um produto interferem na percepção de conforto? Conforto percebido de assentos de trabalho. Revista Estudos em Design, 2002 (no prelo).

HAIR, Joseph F. , ANDERSON, Rolph E., TATHAN, Ronald L., BLACK, William C. Multivariate Data Analysis. New Jersey: Prentice Hall, 5th Ed., 1998.
HELANDER, M. G., ZHANG, L. Field studies of comfort and discomfort in sitting. Ergonomics, v. 40, n. 9, p. 89 915, 1997.

JOHNSON, Richard A., WHICHERN, Dean W. Applied Multivariate Statistical Analysis. New Jersey: Prentice Hall, 3th Ed., 1992.

KRIPPENDORFF, K. Produc Semantics; A Brief Sketch. In: PED Design 2000 Separata das Conferências dos Visitantes Estrangeiros. Novo Hamburgo: Associação de Ensino de Design do Brasil, 2000.

SHACKEL,B., SHIDSEY,K.D., SHIDLEY, P. The Assessment of Chair Comfort. Ergonomics, v. 12 , n.2, p. 269-306, 1969

SHEN, W., PARSONS, K. C. Validity and reliability of rating scales for seated pressure discomfort. International Journal of Industrial Ergonomics, v. 20, p. 441-461, 1997
STRACKER, L. M. Body Discomfort Asssessment Tools In: KARWOWSKI, W., MARRAS, W.S (ed.) The Occupational Ergonomics Handbook. London: CRC, 2000, p. 1239-1252.

LINDEN, Júlio Carlos de Souza van der; KUNZLER, Lizandra Stechman Quintana. A seleção de materiais e o conforto percebido em produtos: investigação da percepção relativa a três materiais utilizados em cadeiras-altas de trabalho. In: VI Congresso Latino-Americano de Ergonomia, XI Congresso Brasileiro de Ergonomia, III Encontro África Brasil de Ergonomia e III Fórum Sul Brasileiro de Ergonomia, ABERGO 2001. 2001, Gramado, RS. Anais do ABERGO 2001. Rio de Janeiro: Associação Brasileira de Ergonomia, 2001.

VERGARA, M., PAGE, A. Relationship between comfort and back posture and mobility in sitting-posture. Applied Ergonomics, v. 33, n. 1, p. 1-8, Jan. 2002.

ZHANG, L., HELANDER, M. G., DRURY, C. G. Identifying Factors of Comfort and Discomfort in Sitting. Human Factors, v. 38, n. 3, p. 377-389, 1996. 


\section{ANEXO 1: Questionário de desconforto}

Utilize as linhas abaixo para indicar a ocorrência de desconforto ou dor, nas diversas regiões de seu corpo, durante a última semana de trabalho (marque com um $\mathrm{X}$ sobre a linha, de acordo com o mapa corporal)

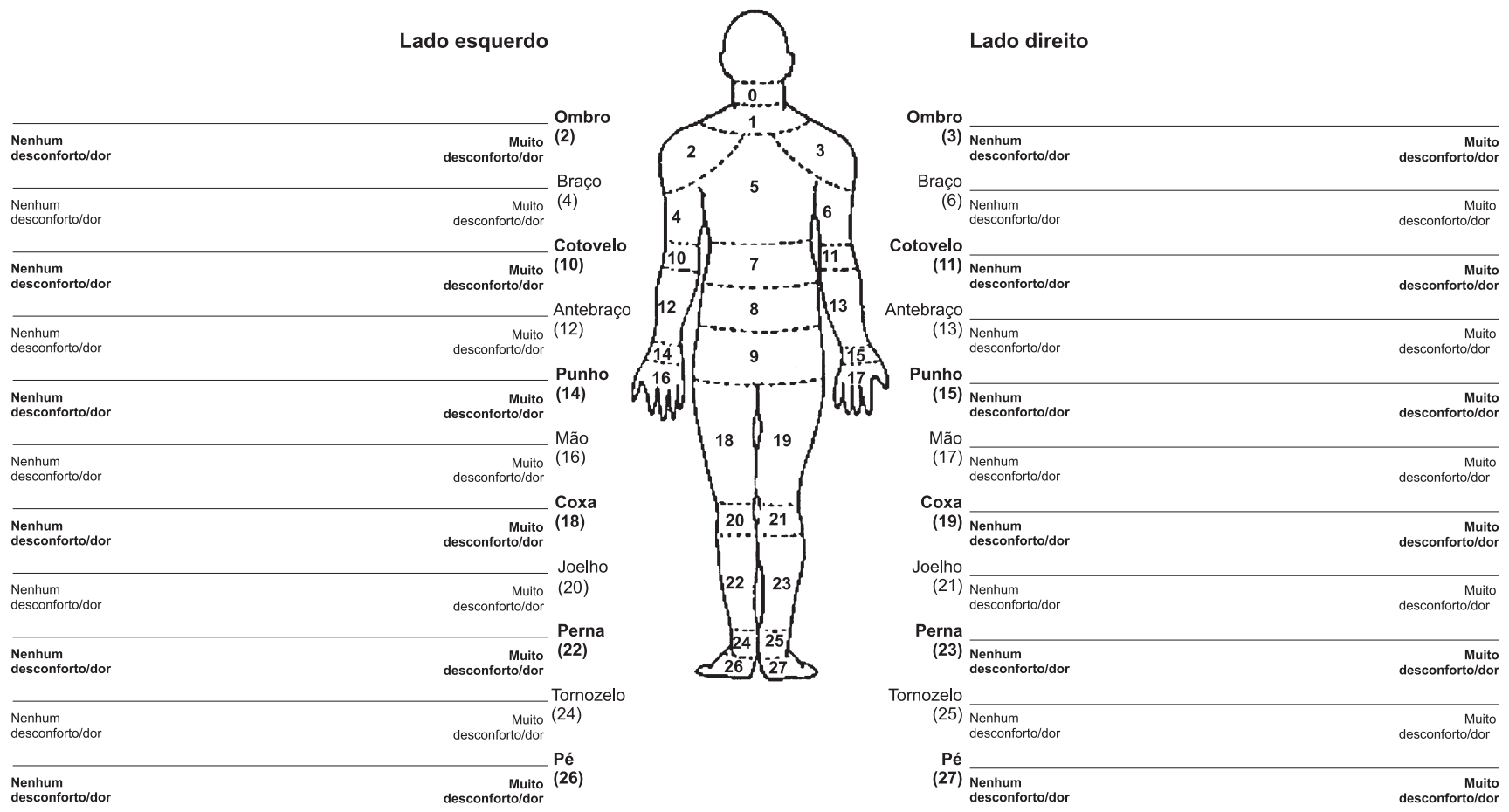

Tronco

\begin{tabular}{|c|c|c|}
\hline \multirow{2}{*}{$\begin{array}{l}\text { Nenhum } \\
\text { desconforto/dor }\end{array}$} & & \multirow{2}{*}{$\begin{array}{l}\text { Pescoço } \\
(0)\end{array}$} \\
\hline & $\begin{array}{r}\text { Muito } \\
\text { desconforto/dor }\end{array}$ & \\
\hline $\begin{array}{l}\text { Nenhum } \\
\text { desconforto/dor }\end{array}$ & $\begin{array}{r}\text { Muito } \\
\text { desconforto/dor }\end{array}$ & $\begin{array}{l}\text { Regiao } \\
\text { cervical(1) }\end{array}$ \\
\hline & & $\begin{array}{l}\text { Costas-superior } \\
\text { (5) }\end{array}$ \\
\hline
\end{tabular}

\begin{tabular}{|c|c|c|}
\hline \multicolumn{3}{|l|}{ Costas-médio } \\
\hline (7) $\begin{array}{l}\mathrm{N} \\
\mathrm{d} d\end{array}$ & $\begin{array}{l}\text { Nenhum } \\
\text { desconforto/dor }\end{array}$ & $\begin{array}{r}\text { Muito } \\
\text { desconforto/dor }\end{array}$ \\
\hline \multicolumn{3}{|l|}{ Costas-inferior } \\
\hline & $\begin{array}{l}\text { Nenhum } \\
\text { desconforto/dor }\end{array}$ & $\begin{array}{r}\text { Muito } \\
\text { desconforto/dor }\end{array}$ \\
\hline Bacia & & \\
\hline & $\begin{array}{l}\text { Nenhum } \\
\text { desconforto/dor }\end{array}$ & $\begin{array}{r}\text { Muito } \\
\text { desconforto/dor }\end{array}$ \\
\hline
\end{tabular}

